\title{
Nasal cytology in children: scraping or swabbing?*
}

\author{
C. Pipolo', S. Bianchini², S. Barberi³, M. Landi', E. D'Auria5', E. Fuccillo', \\ M. Gaffuri', P. Marchisio ${ }^{2}$, C. Rosazza ${ }^{2}$, A.M. Saibene', M. Gelardi ${ }^{7}$, S. Torretta ${ }^{6}$ \\ Rhinology 55: 242-250, 2017 \\ https://doi.org/10.4193/Rhino16.287 \\ 'Otolaryngology Unit, Department of Health Sciences, ASST Santi Paolo e Carlo Hospital, Università degli Studi di Milano, Milan, \\ Italy \\ 2 Pediatric Highly Intensive Care Unit, Department of Maternal and Pediatric Sciences, Fondazione IRCCS Ca' Granda Ospedale \\ Maggiore Policlinico, Università degli Studi di Milano, Milan, Italy \\ ${ }^{3}$ Department of Pediatrics, ASST Fatebenefratelli - Sacco, Milan, Italy \\ ${ }^{4}$ Gruppo pediatrico di cure primarie Asl To1, Turin, Italy \\ ${ }^{5}$ Department of Pediatrics, Department of Health Sciences, ASST Santi Paolo e Carlo Hospital, Università degli Studi di Milano, \\ Milan, Italy \\ ${ }^{6}$ Otolaryngology Unit, Department of Clinical Sciences and Community Health, Fondazione IRCCS Ca' Granda Ospedale Maggiore \\ Policlinico, Università degli Studi di Milano, Milan \\ Otolaryngology Unit, Department of Basic Medical Science, Neuroscience and Sensory Organs, University of Bari Aldo Moro, Bari, \\ Italy \\ *Received for publication: \\ September 30, 2016 \\ Accepted: March 31, 2017
}

\begin{abstract}
Nasal cytology has become a valuable tool in the assessment of a multitude of nasal pathologies in children. Collection methods differ significantly and even though the use of the nasal curette is regarded as the most reliable in adults, most practitioners use the nasal swab in children. However, no studies have validated the reliability and supposed better tolerability of the latter. We have compared these two sampling methods regarding their tolerability and analysed the diagnostic accuracy of the cotton nasal swab (NSW) to identify nasal cytotypes and rhinitis phenotypes, using nasal scraping (NSC) for comparison. In a multicentric prospective study we recruited 208 children and performed nasal cytology by means of NSW and NSC. Microscopic evaluating of the nasal cytotypes was performed and tolerability of NSW and NSC was tested.

Our data revealed a significantly inferior diagnostic accuracy of NSW compared to NSC regarding reliability and cell counts.

Our study is the first to shed light on the role of the sampling tools for pediatric nasal cytology. We documented a poor diagnostic accuracy of NSW, thus suggesting using only the nasal curette in clinical practice. Furthermore, tolerability did not differ between the two, refuting the common thesis that swabs are to be preferred when doing nasal cytology in children.
\end{abstract}

Key words: nasal cytology, eosinophils, rhinitis, allergy, sampling method

\section{Introduction}

Nasal cytology has become a valuable tool in the assessment and diagnosis of a multitude of nasal pathologies. Its aim is to investigate cellular changes and composition in the nasal epithelium and is based on the assumption that healthy nasal mucosa of adults and children alike consists of ciliated-, goblet-, basal- and striated cells and no other cells except, rarely, neutrophils and bacteria. Any detection of inflammatory cells such as eosinophils, mast cells or abundant neutrophils may therefore indicate the presence of an allergic or non-allergic disease. Numerous studies have thus supported the importance of nasal cytology in being able to define pathophysiological mechanisms of acute and chronic disease, the identification of new pathological entities ${ }^{(1,2)}$ and the unmasking of overlapping (allergic and non-allergic) forms ${ }^{(3)}$.

A particular role has been ascribed to nasal cytology in childhood ${ }^{(4,5)}$, partially due to its minimally invasiveness, but even more so because it enables an objective follow-up in this often non-compliant age group. Furthermore, unmasking overlapping allergic and non allergic rhinitis, is valuable in order to achieve possibly more consistent therapeutic results e.g. with allergen immunotherapy ${ }^{(5)}$. 
Over the years, several non-invasive techniques have been developed to sample nasal epithelial cells including nasal washing, suctioning (mucus traps), nasal brushing -packs, -scraping and nasal swabbing. However, only the nasal curette (for nasal scraping) and the nasal swab have become the two methods of choice in modern adult and child nasal cytology, due to their characteristics that render sampling and processing very straightforward and minimally invasive. Even though the nasal curette is widely used in adult nasal cytology, many authors prefer the nasal swab in assessing the paediatric population, as it is perceived to be quicker, less troublesome ${ }^{(6)}$ and better tolerated by children.

While collection with the nasal curette has been shown to produce more reliable results ${ }^{(7)}$ when compared to brushing, and simplifies processing when compared to nasal lavage, no studies have yet validated the sampling by nasal swab in children.

Our aim is therefore to study these two sampling methods in a paediatric population: in a multicentre study we have compared these techniques regarding their tolerability and analysed the diagnostic accuracy of NSW to identify nasal cytotypes and rhinitis phenotypes, using NSC for comparison.

\section{Materials and methods}

Study design and setting

This was a multicentric prospective study of diagnostic accuracy performed in three tertiary hospitals (Centre 1: Department of Otolaryngology and Department of Pediatrics, Santi Paolo e Carlo Hospital, Department of Health Sciences, University of Milan, Milan; Centre 2: Department of Physiopathology and Transplantations of Fondazione IRCCS Ca' Granda Ospedale Maggiore Policlinico, Milan; and Centre 3: Gruppo pediatrico di cure primarie AsI To1, Turin, Italy). The protocol was approved by our local Ethics Committees and was conducted in accordance with the standards of Good Clinical Practice. Written informed consent was obtained from the children's parents or their legal guardians.

\section{Study subjects}

Between 2013 and 2015, we recruited outpatient children aged 3-16 years who were referred to our Departments for either first- and second-level pediatric or otolaryngologic clinical assessment.

The exclusion criteria were the parents' refusal to participate, the use of steroids (intranasal and systemic) for up to 14 days prior to the assessment and the children's inability to cooperate due to pre-existing conditions or non-compliance to nasal examination. In case of recent nasal bleeding (i.e.: occurring in the previous 10 days), the procedure was postponed 7 days after complete recovery.

\section{Interventions}

\section{Clinical assessment}

The demographic and clinical features of each patient were recorded, including their allergic status (as revealed by skin prick tests for the most common classes of aero-allergens carried out within the preceding six months), history of previous or acute airway disease (i.e.: acute otitis media, acute rhinitis, acute pharyngotonsillitis); and history of concomitant systemic diseases. All of the patients underwent an anterior rhinoscopy aimed at evaluating nasal patency, the presence of secretions and the characteristics of nasal mucosa.

\section{Nasal cytology}

Nasal cytology was performed by means of both cotton nasal swab (NSW) in one nostril and by nasal scraping (NSC), using a disposable nasal mucosa curette (Rhino-probe ${ }^{\mathrm{TM}}$, Arlington Scientific, Inc., Springville, UT, USA), in the opposite nostril in every patient. All the children initially underwent NSW, and the first nostril was randomly chosen via a random number generator. The samples were collected during anterior rhinoscopy from the middle portion of the inferior turbinate. The cellular material was placed on a glass slide by rolling the swab/the curette repeatedly, while spreading the material onto the slide, fixed by air drying, stained using the May-Grunwald Giemsa method (Bio Optica Milano S.p.A., Milan, Italy), and then examined under a light microscope. The cells were counted in 10 microscopic fields at $1000 x$ magnification under immersion by the same trained examiner using Meltzer and Jalowayski's semi-quantitative grading (8). Normal smears contain only epithelial cells and rare neutrophils; the pathological rhinitis phenotypes were defined as follows on the basis of cell predominance and clinical features: 1) allergic rhinitis (presence of nasal eosinophils and positive skin prick test for in-season allergen at time of sampling); 2) bacterial rhinitis (presence of bacterial clusters in the sample, accompanied by nasal neutrophils);

3) an eosinophilic form (i.e.: NARES, with nasal eosinophils $>20 \%$ of total cells and the absence of positive skin prick test); 4) a neutrophilic form (i.e.: NARNE, with nasal neutrophils $>50 \%$ of the total cells without any bacterial or fungal element and the absence of positive skin prick test); 5 ) a mast cell form (i.e. NARMA, with nasal mast cells $>10 \%$ of total cells and the absence of positive skin prick test); and 6) a mixed eosinophilic-mast cell form (i.e.: NARESMA, with eosinophils $>20 \%$ and mast cells $>10 \%$ of the total cells and the absence of positive skin prick test) ${ }^{(9,10)}$. The trained clinicians involved in microscopic examination were blinded to the device used for sample collection.

Children reactions including the occurrence of crying, nasal itching, and sneezing after each procedure were recorded; tolerability of NSW and NSC was moreover tested by pain evaluation by means of standardized $0-5$ visual analogue scales (Face pain scale $0=$ no pain; $5=$ maximum pain) or Numerical Rating Scale 
Table 1. Demographic and clinical characteristics of the study population.

\begin{tabular}{|c|c|c|}
\hline & Characteristics & No. $(\%)$ \\
\hline No. of males & & $141(67.8)$ \\
\hline \multirow[t]{4}{*}{ Collecting centre } & & $7.6 \pm 2.9$ \\
\hline & No. of pts. from centre 1 & $85(40.9)$ \\
\hline & No. of pts. from centrer 2 & $98(47.1)$ \\
\hline & No. of pts. from centre 3 & $25(12.0)$ \\
\hline \multirow[t]{10}{*}{ No. of pts. with history of previous airway disease } & None & $15(7.2)$ \\
\hline & Recurrent or chronic middle ear infections & $57(27.4)$ \\
\hline & Recurrent or chronic upper airway infections & $76(36.5)$ \\
\hline & Recurrent or chronic lower airway infections & $42(20.2)$ \\
\hline & Other diseases & $18(8.7)$ \\
\hline & None & $150(72.1)$ \\
\hline & Acute otitis media & $3(1.4)$ \\
\hline & Acute rhinitis & $44(21.1)$ \\
\hline & Acute pharyngotonsillitis & $2(1.0)$ \\
\hline & Other diseases & $9(4.4)$ \\
\hline No. of pts. with allergy & & $119(57.2)$ \\
\hline
\end{tabular}

No.: number; sd: standard deviation; pts.: patients.

(NRS), in children older than 8 years ${ }^{(11)}$.

\section{Sample size}

Sample size was calculated on the basis of the primary endpoint, which was to compare diagnostic accuracy of NSW to the gold standard diagnostic technique (i.e.: NSC) for nasal cytology in identification of pathological rhinitis phenotypes. We assumed that sensitivity of NSC was $92 \%$; a sample size of 134 children thus achieved $80 \%$ power to detect a difference between the two diagnostic tools' sensitivity of about $7 \%$.

\section{Statistical analysis}

Descriptive statistics were used to describe the population basic characteristics, the cytological features, and tolerability of NSW and NSC. The ordinal variables were expressed as mean values and their standard deviations. Tolerability was compared by means of Fisher's exact probability test or Wilcoxon signed rank sum test.

Diagnostic accuracy of NSW compared to NSC in detection of specimens adequate to microscopic lecture, pathological rhinitis phenotypes, peculiar nasal cytotypes, and nasal cells was tested by means of receiver-operating characteristic (ROC) analysis, and by computing its sensitivity, specificity, positive (LR+) and negative (LR-) likelihood ratios, and the area under the receiveroperating characteristic curve (AUC), with its $95 \%$ confidence intervals ( $95 \% \mathrm{Cls})$.

The concordance of the two diagnostic tools in identifying the same rhinitis phenotypes was tested and expressed as the per- centage of accordant diagnosis.

The effect of potential confounders such as the collecting centre, the presence of allergy or any acute airway disease was moreover tested by means of dedicated subgroups analysis. Statistical significance was set at $p$-value $<0.05$. The data were analysed using STATA 10.0 software (StataCorp, College Station, TX, USA).

\section{Results}

The final analysis was based on the grounds of 208 children $(141 / 208,67.8 \%$ males; mean age $=7.6 \pm 2.9$ years $)$. Six children were not included due to pre-existing conditions or non-compliance to anterior nasal examination (2/6 Autism Spectrum Disorder, 4/6 non compliance). Demographic and clinical characteristics are reported in Table 1. Pathological nasal specimens were detected in about $25 \%$ of cases by NSW and in about $32 \%$ of cases by NSC ( $p$-value $=$ not significant - n.s.-), and allergic rhinitis resulted to be the most frequent pathological rhinitis phenotype in both of them (discovered respectively in $55.4 \%$ of pathological samples collected by NSW, and in $50.0 \%$ of those collected by NSC; $p$-value=n.s.). About $29 \%$ and $15 \%$ of specimens respectively collected by NSW and NSC resulted to be inadequate for microscopic lecture ( $p$-value $<0.001)$. Detailed cytological characteristics are reported in Table 2.

When diagnostic accuracy of NSW (in detection of: adequate specimens, pathological rhinitis phenotypes, peculiar nasal cytotypes, and nasal cells) was tested, sensitivity varied between $16.7 \%$ and $98.5 \%$, and specificity was not higher than $88.6 \%$ for 
Table 2. Cytological findings at NSW and NSC.

\begin{tabular}{|c|c|c|c|c|}
\hline Characteristics & & NSW & NSC & p-value \\
\hline No. of specimens inadequate for lecture & & $61(29.3)$ & $31(15)$ & $<0.001$ \\
\hline No. of specimens with pathological rhinitis phenotypes & & $52(25.0)$ & $67(32.4)$ & n.s. \\
\hline \multirow[t]{7}{*}{ Pathological rhinitis phenotypes } & AR & $46(55.4)$ & $41(50.0)$ & n.s. \\
\hline & Bacterial rhinitis & $22(26.5)$ & $23(28.0)$ & n.s. \\
\hline & NARES & $5(6.0)$ & $6(7.3)$ & n.s. \\
\hline & NARNE & $3(3.6)$ & $3(3.6)$ & n.s. \\
\hline & NARMA & $5(6.0)$ & $5(6.1)$ & n.s. \\
\hline & NARESMA & $0(0.0)$ & $2(2.5)$ & n.s. \\
\hline & Other & $2(2.5)$ & $2(2.5)$ & n.s. \\
\hline \multirow[t]{4}{*}{ No. of specimens with cells } & Bacteria & $26(12.5)$ & $42(20.2)$ & 0.034 \\
\hline & Eosinophils & 45 (21.6) & $54(26.0)$ & n.s. \\
\hline & Neutrophils & $105(50.5)$ & 159 (76.4) & $<0.001$ \\
\hline & Mast cells & 31 (14.9) & $30(14.4)$ & n.s. \\
\hline
\end{tabular}

NSW: nasal swab; NSC: nasal scraping; n.s.: not significant; AL: allergic rhinitis; NARES: non allergic rhinitis with nasal eosinophils; NARNE: non allergic rhinitis with nasal neutrophils; NARMA: non allergic rhinitis with nasal mast cells; NARESMA: non allergic rhinitis with nasal eosinophils and mast cells.

Table 3. Measures of diagnostic accuracy of NSW compared to NSC.

\begin{tabular}{|c|c|c|c|c|}
\hline Outcomes & & Sensitivity, \% & Specificity, \% & LR+, \% \\
\hline Identification of adequate specimens & & 35.5 & 71.6 & 1.3 \\
\hline Identification of pathological rhinitis phenotypes & & 37.3 & 81.4 & 2.0 \\
\hline \multirow[t]{7}{*}{ Identification of pathological rhinitis phenotypes } & AR & 78.4 & 24.4 & 1.0 \\
\hline & Bacterial rhinitis & 90.3 & 8.7 & 1.0 \\
\hline & NARES & 98.0 & 16.7 & 1.2 \\
\hline & NARNE & 98.5 & 0.0 & 1.0 \\
\hline & NARMA & 98.0 & 20.0 & 1.2 \\
\hline & NARESMA & - & - & - \\
\hline & Other & 97.6 & 0.0 & 1.0 \\
\hline \multirow[t]{4}{*}{ Nasal cells identification } & Bacteria & 16.7 & 88.6 & 1.5 \\
\hline & Eosinophils & 37.0 & 83.8 & 2.3 \\
\hline & Neutrophils & 52.8 & 57.1 & 1.2 \\
\hline & Mast cells & 26.7 & 87.1 & 2.1 \\
\hline
\end{tabular}

AL: allergic rhinitis; NARES: non allergic rhinitis with nasal eosinophils; NARNE: non allergic rhinitis with nasal neutrophils; NARMA: non allergic rhinitis with nasal mast cells; NARESMA: non allergic rhinitis with nasal eosinophils and mast cells; LR+: positive likelihood ratio; LR-: negative likelihood ratio; AUC: area under the receiver-operating characteristic curve; $95 \% \mathrm{Cl}$ : $95 \%$ confidence interval.

all the outcomes (Table 3). This resulted in a substantial inaccuracy of NSW, as attested by the AUCs, ranging between 0.49 and $0.60{ }^{(12)}$ (Table 3 and Figure 1). These data were confirmed by testing the concordance of NSW with NSC in identifying the same nasal cytotype, which resulted $51.4 \%$.

Subgroups analysis performed in order to test if the presence of allergy (AUC for the absence of allergy $=0.54,95 \% \mathrm{Cl}=0.41-0.66$; AUC for the presence of allergy $=0.54,95 \% \mathrm{Cl}=0.40-0.68 ; \mathrm{p}$ value $=$ n.s.) or any acute airway disease (AUC for the absence of acute airway disease $=0.50,95 \% \mathrm{Cl}=0.40-0.61$; AUC for the presence of acute airway disease $=0.63,95 \% \mathrm{Cl}=0.42-0.83$; $\mathrm{p}$-value $=$ n.s.) could act as confounders on diagnostic accuracy of NSW in 


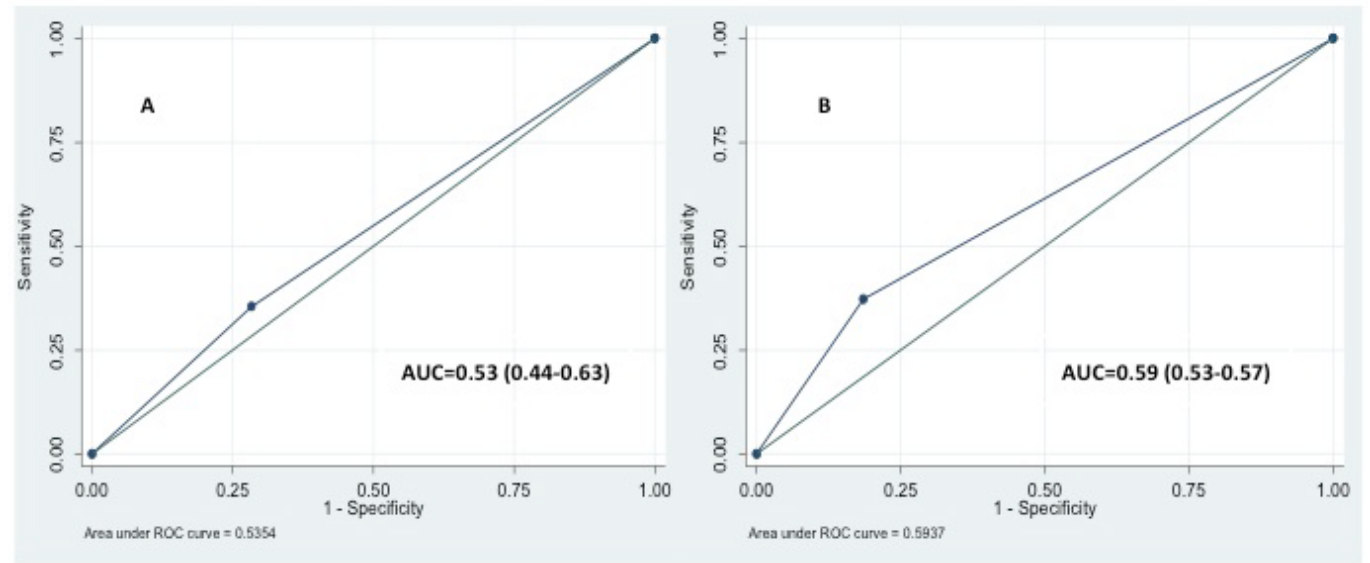

Figure 1. ROC curve for identification of adequate specimens (A) and identification of pathological nasal cytotypes (B). ROC: receiver-operating characteristic; AUC: area under the receiver-operating characteristic curve.
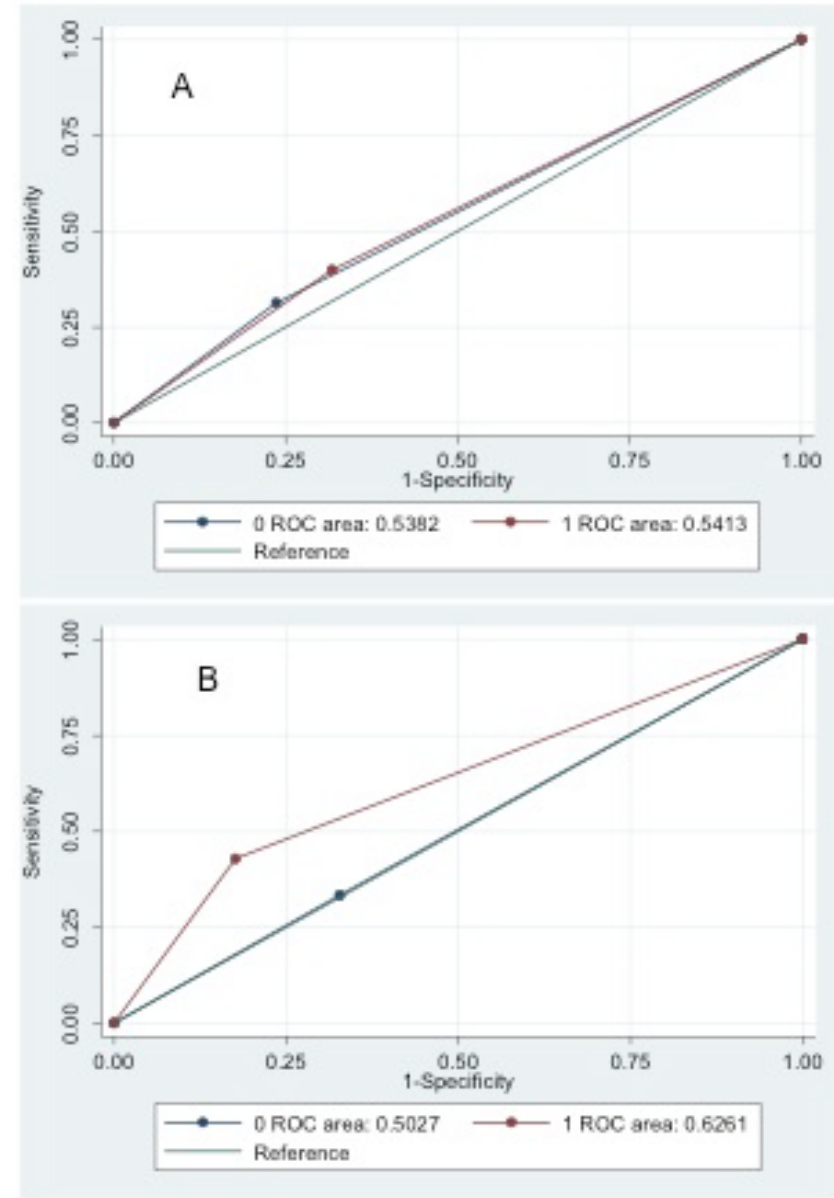

Figure 2. ROC curve for identification of adequate specimens according to the presence of allergy $\left(A\right.$, blue line $=$ no allergy, red line $=$ allergy ${ }_{s} p-$ value $=n . s$. $)$ and acute airway disease $(B$, blue line $=$ no acute airway disease, red line= acute airway disease; $p$-value= $=$.s.). The collecting centre was not tested as a confounder due to numerical incongruity. ROC: receiver-operating characteristic; AUC: area under the receiver-operating characteristic curve; n.s.: not significant. identification of adequate specimens did not document any statistically significant difference in the AUCs (Figure 2). Moreover, the collecting centre (AUC for centre $1=0.60,95 \% \mathrm{Cl}=0.45-0.74$; AUC for centre $2=0.51,95 \% \mathrm{Cl}=0.41-0.60$; AUC for centre $3=$ $0.52,95 \% \mathrm{Cl}=0.31-0.72 ; \mathrm{p}$-value $=$ n.s.), the presence of allergy (AUC for the absence of allergy $=0.53,95 \% \mathrm{Cl}=0.42-0.63$; AUC for the presence of allergy $=0.64,95 \% \mathrm{Cl}=0.55-0.73 ; \mathrm{p}$-value $=n . s$.) or any acute airway disease disease (AUC for the absence of acute airway disease $=0.57,95 \% \mathrm{Cl}=0.48-0.65 ; \mathrm{AUC}$ for the presence of acute airway disease $=0.60,95 \% \mathrm{Cl}=0.48-0.72 ; \mathrm{p}$-value $=$ n.s.) did not statistically influence diagnostic accuracy of NSW in the identification of pathological rhinitis phenotypes, as resulted by the AUCs comparison (Figure 3).

Both procedures were well tolerated, as attested by mean VAS pain values (respectively $1.9 \pm 1.5$ after NSW, and $1.8 \pm 1.6$ after NSC; -value $=$ n.s.), and by the occurrence of crying (reported respectively in $35.6 \%$ of children after NSW, and in $28.8 \%$ of children after NSC; $\mathrm{p}$-value $=$ n.s.). Furthermore, only minor local and transient side effects were documented after sampling, with nasal itching being the most frequent (reported respectively in $63.5 \%$ of children after NSW, and in $63.9 \%$ of children after NSC; $\mathrm{p}$-value $=$ n.s.). No major side effects such as bleeding occurred. Tolerability of NSW was comparable to tolerability of NSC, as no statistically significant differences between the procedures were found when mean VAS /NRS pain values, the occurrence of crying, nasal itching or sneezing were tested (Table 4). When subgroups analysis was performed in order to test if the collecting centre, the presence of allergy or any acute airway disease could act as confounders, we did not find any statistically significance in itching, sneezing and crying. However, we found that mean pain measured with VAS was higher in NSW compared to NSC in center $2(2.3 \pm 1.6$ vs. $1.6 \pm 1.4$; p-value $=$ $0.004)$ and $3(1.1 \pm 0.6$ vs. $0.5 \pm 0.4 ; p$-value $<0.001)$, and that it 

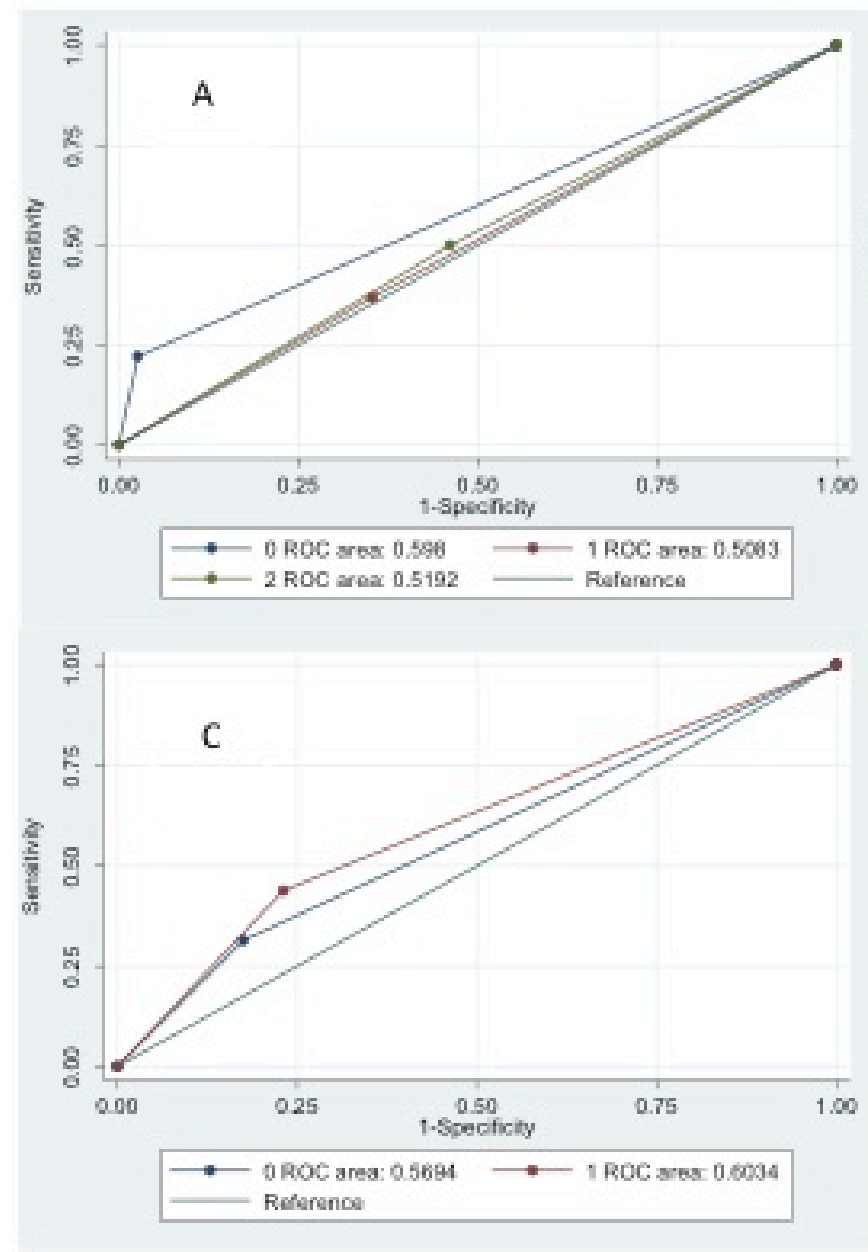

Figure 3. ROC curve for identification of pathological nasal cytotypes according to the collecting centre $(A$, blue line $=$ centre 1 , red line $=$ centre 2 , green line $=$ centre $3 ; p$-value $=$ n.s.), the presence of allergy $(B$, blue line $=$ no allergy, red line $=$ allergy ${ }_{s} p$-value $=n$.s.) and acute airway disease $(C$, blue line $=$ no acute airway disease, red line $=$ acute airway disease; p-value $=$ n.s.). ROC: receiver-operating characteristic; AUC: area under the receiver-operating characteristic curve; n.s.: not significant.

Table 4. Tolerability of NSW and NSC.

\begin{tabular}{|lccc|}
\multicolumn{1}{c}{ Variable } & NSW & NSC & p-value \\
\hline Crying (\%) & $74(35.6)$ & $60(28.8)$ & 0.08 \\
\hline Nasal itching (\%) & $132(63.5)$ & $133(63.9)$ & 0.37 \\
\hline Sneezing (\%) & $38(18.3)$ & $37(17.8)$ & 0.90 \\
\hline Mean VAS pain \pm sd & $1.9(1.5)$ & $1.8(1.6)$ & 0.37
\end{tabular}

NSW: nasal swab; NSC: nasal scraping; VAS: visual analogue scale; sd: standard deviation; n.s.: not significant.

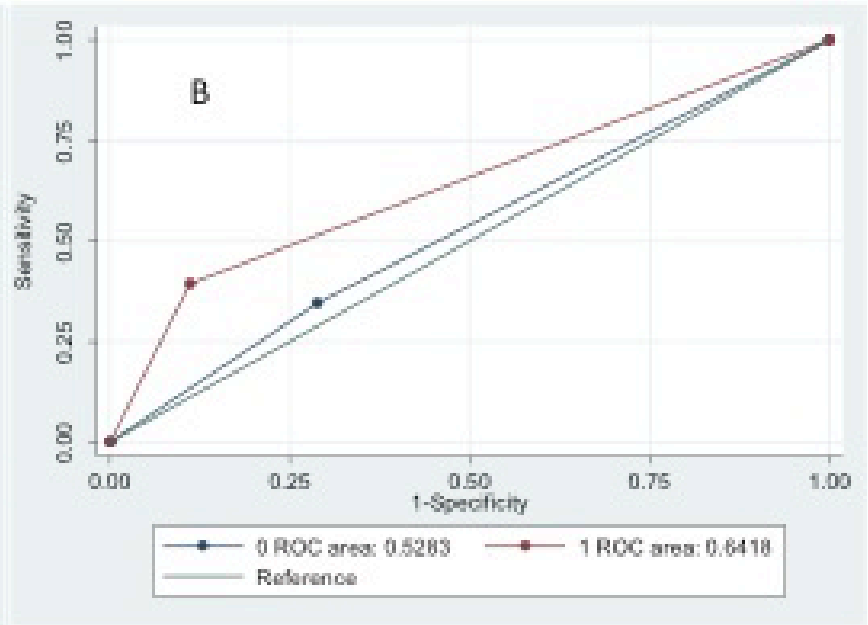

was higher in NSC compared to NSW in centre 1 ( $2.4 \pm 1.6$ vs. $1.8 \pm 1.5 ; p$-value $=0.004$ ). No other confounders were found to significantly influence NSW and NSC tolerability (Table 5).

\section{Discussion}

To the best of our knowledge this is the first study evaluating diagnostic accuracy of NSW in assessing nasal cytotypes in children. NSC was chosen as the comparing sampling technique because even if different sampling methods have been proposed (including blowing the nose, nasal brushing, and direct biopsy) ${ }^{(4,13-15)}$, NSC still represents the tool of choice ${ }^{(18,19)}$ given that it was found to be more accurate than both cotton swab and brushing in adult population ${ }^{(20)}$ and it is supposed to be well tolerated by the paediatric population, too. However, we have to take into account that NSC is not a nasal biopsy and as such could perhaps over or underestimate nasal pathology. We found that NSW achieves adequate sample collection in a significantly lower number of patients compared to NSC, as nearly one third of NSW-determined specimens resulted to be inadequate for microscopic lecture, thus impeding diagnosis in a not negligible subset of patients.

When ROC analysis was performed in order to evaluate diagnostic accuracy of NSW compared to NSC in detecting adequate specimens, the presence of pathological rhinitis phenotypes, and in discriminating peculiar nasal cytotypes, acceptable values in sensitivity and specificity were not simultaneously found for any of the assessed outcome. This results in corresponding AUCs ranging between 0.49 and 0.60 , thus suggesting a substantial poor diagnostic accuracy of NSW compared to NSC. This finding was confirmed when subgroup analysis performed according to some possible confounders (such as the collecting centre, the presence of allergy or any acute upper airway disease) was conducted. The global poor diagnostic accuracy of NSW was moreover suggested by the concordance test, which showed that NSW was able to correctly identify nasal cytological profiles only in about $50 \%$ of cases. 
Table 5. Analysis of tolerability by possible confounders.

\begin{tabular}{|c|c|c|c|c|c|}
\hline \multirow[t]{7}{*}{ Crying (\%) \pm sd } & Centre & Centre 1 & $45.9 \pm 5.4$ & $47.1 \pm 5.4$ & n.s. \\
\hline & & Centre 2 & $21.4 \pm 4.1$ & $30.6 \pm 4.6$ & n.s. \\
\hline & & Centre 3 & $33.6 \pm 3.9$ & $29.8 \pm 5.1$ & n.s. \\
\hline & Acute airway infection & Yes & $19.0 \pm 5.1$ & $31.0 \pm 6.1$ & n.s. \\
\hline & & No & $32.7 \pm 3.8$ & $37.3 \pm 3.4$ & n.s. \\
\hline & Allergy & Yes & $31.1 \pm 4.2$ & $37.0 \pm 4.4$ & n.s. \\
\hline & & No & $25.8 \pm 4.6$ & $33.7 \pm 5.0$ & n.s. \\
\hline \multirow[t]{7}{*}{ Nasal itching $(\%) \pm s d$} & Centre & Centre 1 & $75.3 \pm 4.7$ & $65.9 \pm 5.1$ & n.s. \\
\hline & & Centre 2 & $47.0 \pm 5.0$ & $52.0 \pm 5.0$ & n.s. \\
\hline & & Centre 3 & $63.5 \pm 4.8$ & $61.9 \pm 4.7$ & n.s. \\
\hline & Acute airway infection & Yes & $63.8 \pm 6.3$ & $75.9 \pm 5.6$ & n.s. \\
\hline & & No & $64.0 \pm 3.9$ & $58.7 \pm 4.0$ & n.s. \\
\hline & Allergy & Yes & $66.4 \pm 4.3$ & $67.2 \pm 4.3$ & n.s. \\
\hline & & No & $60.1 \pm 5.2$ & $58.4 \pm 5.2$ & n.s. \\
\hline \multirow[t]{7}{*}{ Sneezing (\%) \pm sd } & Centre & Centre 1 & $8.2 \pm 3.0$ & $14.1 \pm 3.8$ & n.s. \\
\hline & & Centre 2 & $11.2 \pm 3.2$ & $5.1 \pm 2.2$ & n.s. \\
\hline & & Centre 3 & $23.5 \pm 4.3$ & $22.9 \pm 4.5$ & n.s. \\
\hline & Acute airway infection & Yes & $36.2 \pm 6.3$ & $32.7 \pm 6.2$ & n.s. \\
\hline & & No & $11.3 \pm 2.6$ & $12.0 \pm 2.6$ & n.s. \\
\hline & Allergy & Yes & $21.0 \pm 3.7$ & $21.8 \pm 3.8$ & n.s. \\
\hline & & No & $14.6 \pm 3.7$ & $12.3 \pm 3.5$ & n.s. \\
\hline \multirow[t]{7}{*}{ Mean VAS pain $\pm \mathrm{sd}$} & Centre & Centre 1 & $1.8 \pm 1.5$ & $2.4 \pm 1.6$ & 0.004 \\
\hline & & Centre 2 & $2.3 \pm 1.6$ & $1.6 \pm 1.4$ & 0.004 \\
\hline & & Centre 3 & $1.1 \pm 0.6$ & $0.5 \pm 0.4$ & $<0.001$ \\
\hline & Acute airway infection & Yes & $1.6 \pm 1.5$ & $1.5 \pm 1.5$ & n.s. \\
\hline & & No & $1.9 \pm 1.4$ & $1.9 \pm 1.6$ & n.s. \\
\hline & Allergy & Yes & $1.8 \pm 1.6$ & $1.9 \pm 1.6$ & n.s. \\
\hline & & No & $2.1 \pm 1.6$ & $1.6 \pm 1.5$ & n.s. \\
\hline
\end{tabular}

NSW: nasal swab; NSC: nasal scraping; VAS: visual analogue scale; sd: standard deviation; n.s.: not significant.

These results may be related to the fact that curette-probe sampling probably involves a deeper cell collection into the nasal mucosa due to the scraping process, or that some cells may be entrapped into the cotton swab, thus avoiding complete deposition of harvested material onto the slide. Whatever the reason, this results in a poor cellularity of swab-determined samples, which is responsible for both the high rate of NSWobtained specimens inadequate for microscopic lecture and the substantial inaccuracy of NSW in achieving reliable nasal cytolo- gical profiles. Our results support this hypothesis, given that the percentage of specimens with either bacterial or inflammatory cells was lower among NSW-obtained samples compared to NSC-obtained samples, as documented in Table 2. The poor capability of NSW in detecting nasal cells results in its inaccuracy in discriminating between physiologic and pathologic nasal cytotypes and among peculiar pathological nasal profiles. When the tolerability of the two procedures was assessed, our data showed that both of them were well-tolerated, as resulted 
by VAS /NRS pain analysis, and the absence of any major side effects. As a matter of fact, only a slight and transient discomfort was documented by the occurrence of crying respectively in $36 \%$ and $29 \%$ of children undergoing NSW and NSC, and the occurrence of nasal itching and sneezing. Moreover, our results seem to question the common opinion of less invasiveness of NSW, as attested by the absence of any statistically significant difference in tolerability between NSW and NSC. However, VASpain analysis adjusted for collecting centre showed that pain may be considered operator dependent and that both tools can be associated with more or less pain, albeit in two out of three centres NSC was considered less painful than NSW.

In our opinion the possible explanation for these phenomena stands in the different conformation of the two devices: even if the NSW presents a softer cotton tip than the NSC, the plastic curette results stiffer than NSW and is therefore easier to maneuver towards the middle part of the inferior turbinate for a faster and more precise sampling, without touching the extremely innervated nasal septum.

Our results are not directly comparable with previous ones, as the only one study assessing diagnostic accuracy of NSW compared to NSC only focused on the identification of the eosinophilic pattern in allergic adults ${ }^{(20)}$ and did not evaluate the tolerability of the tools. However, our data is in line with results by Lin et al. ${ }^{(20)}$, which showed that the curette-probe determined nasal eosinophil quantitation had greater area under the ROC curves (and therefore a better diagnostic accuracy in detecting patients with allergic rhinitis) than the cotton swab-obtained one, when definition of allergic rhinitis was performed on the basis of elevated aero-allergen specific IgE and either significant nasal mucosal appearance abnormalities or a referring physician's diagnosis of rhinitis.

A limitation of this study could be the diagnosis of the different rhinitis phenotypes and especially NARES without testing for nasal allergen-specific lgE, in order to differentiate NARES from local allergic rhinitis. However, our aim of the study was to compare cytological sampling methods that are commonly used and we therefore did not include this testing believing it would not change our conclusions.
Our results documenting a poor diagnostic accuracy of NSW in determining nasal profiles and a comparable tolerability of NSC and NSW in children can be directly transferred to the clinical practice and are completely reproducible, given that they were substantially overlapping among three different centres and none of the variables assessed as possible confounders resulted to be significant.

\section{Conclusion}

Our study is the first to shed light on the role of the tools used for pediatric nasal cytology collection regarding accuracy and tolerability. We documented a substantial poor diagnostic accuracy of NSW compared to NSC in determining nasal profiles, thus suggesting to limit the use to NSC only in clinical practice. Furthermore, tolerability did not differ between the two, refuting the common thesis that swabs are to be preferred when doing nasal cytology in children. Interestingly however, reported pain (although minimal) did indeed differ between operators, underlining the importance of a correct training.

\section{Authorship contribution}

CP: Has conceived the study, analysed the nasal samples, written the manuscript; SBi: Has done the sampling and recruiting of the children at the Fondazione IRCCS Ca' Granda Ospedale Maggiore Policlinico; SBa: Has done the sampling and recruiting of the children at ASST Santi Carlo e Paolo; ML: Has done the sampling and recruiting of the children at the ASL 1 Turin and critically reviewed the manuscript; ED'a: Has done the recruiting of the children at ASST Santi Carlo e Paolo; EF: Has done the sampling and the follow-up of the patients at ASST Santi Carlo e Paolo; MG: Has written the discussion; PM: Has conceived the study; CR: Has read the samples of the Fondazione IRCCS Ca' Granda Ospedale Maggiore Policlinico; AMS: Has recruited the patients and reviewed the article; MG: Has read the samples of ASST Santi Carlo e Paolo and critically revised the manuscript; ST: Has done the statistical analysis and made the tables.

\section{Conflict of interest}

The authors have no potential conflict of interests concerning this paper and they do not have financial disclosures to make.

\section{References}

1. Jacobs RL, Freedman PM, Boswell RN Nonallergic rhinitis with eosinophilia (NARES syndrome). Clinical and immunologic presentation. J Allergy Clin Immunol. $1981 ; 67(4): 253-62$

2. Gelardi M, Maselli del Giudice A, Fiorella $\mathrm{ML}$ et al. Non-allergic rhinitis with eosinophils and mast cells constitutes a new severe nasal disorder. Int J Immunopathol Pharmacol. 2008;21(2):325-31

3. Gelardi M, Russo C, Fiorella ML, Fiorella R,
Canonica GW, Passalacqua G. When allergic rhinitis is not only allergic. Am J Rhinol Allergy. $2009 ; 23(3): 312-5$.

4. Jirapongsananuruk $\mathrm{O}$, Vichyanond P. Nasal cytology in the diagnosis of allergic rhinitis in children. Ann Allergy Asthma Immunol. 1998;80(2):165-70

5. Gelardi M, Luigi Marseglia G, Licari A et al. Nasal cytology in children: recent advances. Ital J Pediatr. 2012; 25;38:51

6. Gelardi M, Fiorella ML, Leo G, Incorvaia C. Cytology in the diagnosis of rhinosinusi- tis. Pediatr Allergy Immunol. 2007;18 Suppl 18:50-2.

7. Lin RY1, Nahal A, Lee $M$, Menikoff $H$. Cytologic distinctions between clinical groups using curette-probe compared to cytology brush. Ann Allergy Asthma Immunol. 2001;86(2):226-31.

8. Meltzer EO, Jalowaysky AA. Nasal cytology in clinical practice. Am J Rhinol 1988; 2:4754

9. Ellis AK, Keith PK. Nonallergic rhinitis with eosinophilia syndrome and related disor- 
ders. Clin Allergy Immunol. 2007;19:87-100

10. Torretta S, Cappadona M, Cairoli D Pignataro L. Airborne nitric oxide and nasal cytology in patients with chronic rhinosinusitis and nasal polyps. J Biol Regul Homeost Agents. 2015;29(4):969-76.

11. Hicks $C L$, von Baeyer $C L$, Spafford $P A$, van Korlaar I, Goodenough B. The Faces Pain Scale-Revised: toward a common metric in pediatric pain measurement. Pain 2001;93(2):173-83.

12. Swets J.A. 1998 Measuring the accuracy of diagnostic systems. Science, 240, 1285-93

13. Jean R, Delacourt C, Rufin P et al. Nasal cytology in rhinitis children: comparison between brushing and blowing the nose. Allergy. 1996;51(12):932-4.

14. Pastorello EA, Riario-Sforza GG, Incorvaia C, Segala M, Fumagalli M, Gandini R. Comparison of rhinomanometry, symptom score, and inflammatory cell counts in assessing the nasal late-phase reaction to allergen challenge. J Allergy Clin Immunol. 1994;93(1 Pt 1):85-92.

15. Howarth PH. The cellular basis for allergic rhinitis. Allergy. 1995;50(23 Suppl):6-10.

16. Cohen GA, MacPherson GA, Golembesky HE, Jalowayski AA, O'Connor RD. Normal nasal cytology in infancy. Ann Allergy. 1985;54(2):112-4.

17. Basibuyuk T, Ozaydin E, Cengizlier R. Nasal cytology in the diagnosis and treatment of sinusitis in atopic and nonatopic children. Otolaryngol. 2006;35(4):255-60.

18. Ronchetti R, Villa MP, Martella $S$ et al Nasal cellularity in 183 unselected schoolchildren aged 9 to 11 years. Pediatrics. 2002;110(6):1137-42.

19. Gibson PG, Stuart JE, Wlodarczyk J, Olson LG, Hensley MJ. Nasal inflammation and chronic ear disease in Australian Aboriginal children. J Paediatr Child Health.
1996;32(2):143-7.

20. Lin RY, Clarin E, Lee M, Menikoff $H$, Nahal A. Patterns of nasal eosinophilia in allergy clinic patients as determined by swab and curette sampling. Allergy Asthma Proc. 1997;18(4):221-6.

\section{Carlotta Pipolo}

Via Antonio di Rudinì 8

20142 Milano

Italy

Tel: +39-2-8184 4249

E-mail: carlotta.pipolo@unimi.it 